cord neuroanatomy appears to be similar in the two species, and electrophysiological experiments (impossible in C. elegans) have led to the formulation of a model for bend formation in Ascaris. The same two classes of acetylcholinesterase are found in both nematodes, and both are widely distributed in Ascaris (C. Johnson, University of Wisconsin, Madison). The elimination by mutation of the two classes (but not either class alone) leads to defective movement in C. elegans (J. Culotti, Göttingen).

The participants seemed to be united in their enthusiasm for $C$. elegans, and their interest in all aspects of its biology. Specialisation has not led to fragmentation.

\section{Nonsolar planets and their detection}

\section{from David W. Hughes}

I AM never sure which is the more surprising supposition - that our planetary system is of such extreme rarity that it might be unique in the Galaxy - or that planetary systems similar to ours exist in orbit around one in every four stars. There seems to be no inherent reason why Earth should not be the only home for living creatures, an assumption that obviously makes our search for extraterrestrial life futile. On the other hand planets may well be common. There seems to be no sharp distinction between binary and multiple star systems and stars with planetary companions. The masses of small stars in binary systems seemingly grade continuously down to the masses of planets. The obvious solution to this dilemma is 'look and see'. The discovery of nonsolar planets would provide a vital clue to the origin of the solar system, a subject which is bedevilled at the present by the fact that only one solar system can be studied in detail. Bracewell and MacPhie have reviewed the problems inherent in searching for nonsolar planets in a recent edition of Icarus $(38,136 ; 1979)$.

Astrometry is one of the existing techniques which, according to the authors, "with sufficient refinement may detect nonsolar planets". Plate scales of 15 arc sec per mm can be obtained with very long focus refracting telescopes and these enable very small angular displacements of stellar images to be detected. A year's observation gives a precision of 0.003 arc sec. The problem is exemplified by the fact that an observer of the Sun from a distance of 10 parsec would be looking for an epicycloid wiggle of amplitude about 0.0005 arc sec and period of about $12 \mathrm{yr}$ just to detect Jupiter. Needless to say, many researchers regard the astrometric technique as already sufficiently refined to detect planets. Van de Kamp and
Lippincott of the Sproul Observatory have reported the discovery of invisible planets around Barnard's star, Epsilon Eridani and $\mathrm{Ci} 2354$. Lalande 21185 is also thought to have a planetary companion. It is interesting to note however that unlike the near circular orbits of our planets these objects seem to have high eccentricities, a factor they have in common with double stars.

Orbiting planets can also produce a sinusoidal variation in the stellar radial velocity. Observed in the ecliptic plane and from a distance, the amplitude of the variation for the Sun is $12 \mathrm{~ms}^{-1}$, equivalent to a $3 \times 10^{-5} \mathrm{~nm}$ shift in the $656.28 \mathrm{~nm}$ Fraunhofer line of the solar spectrum. Present practice achieves a precision of several hundred metres per second so this is a technique for the future.

When the combined light of Jupiter and the Sun is viewed from say 10 parsec only about one in $6 \times 10^{8}$ photons received actually comes from Jupiter. Also light from the Sun, diffracted by the edge of the telescope aperture obscures the planetary light. Diffraction may be overcome by shading the aperture, a process known as apodisation in which light transmission falls off gradually from the centre of the field towards the edge. Such a telescope, in orbit above the atmosphere can theoretically detect planets.

Infrared long baseline interferometry can be used. Planetary thermal infrared radiation might not be strong but at least it comes from near the peak of the spectral distribution. For stars, the infrared spectral region is well down from the peak of the spectrum. The ratio between stellar power and planetary power drops to around 5,000 at wavelengths longer than about $20 \mu \mathrm{m}$. As the distance between the elements of an interferometer is increased, fewer and fewer objects can be detected. The ones that can are those with the smallest angular diameter and this of course favours planets. Bracewell and MacPhie consider possible infrared observations that can be obtained by future space probes. The first one entails having two orbiting infrared ( $40 \mu \mathrm{m}$ say) collectors separated by $44 \mathrm{~km}$ (that is $10^{9}$ wavelengths). The angular diameter of the star is then about five times the interference fringe spacing so as the star changes its position relative to the fringe pattern the receiver power hardly varies. The planet however is about one tenth the stellar angular diameter and fringes sweeping over the planet produce a substantial modulation. Unfortunately the observing advantage gained is only about 20 , which does little to reduce the previous factor of 5,000 .

An improvement can be made by placing a minimum of the interference pattern on the star. The planet is then placed at the

David W. Hughes is a Lecturer in the Department of Physics, University of Sheffield. adjacent maximum, this requiring, at $40 \mu \mathrm{m}$, a base line of $7.7 \mathrm{~m}$ for an angular separation of $2.6 \times 10^{-6}$ rad between star and planet. The star to planet power ratio drops to an amazing $1 / 80$. This signal can be modulated, and thus more easily detected against the background, by spinning the interferometer about an axis through the star. Unfortunately the basic pointing accuracy required is $0.001 \mathrm{sec}$ arc. For a detector of area $1 \mathrm{~m}^{2}$ and a receiver of bandwidth $\Delta \lambda$ equal to $0.1 \lambda$ a planet like Jupiter 10 parsec away would produce a flux of 2 photons per second. A reasonable signal to noise ratio is obtained with $10 \mathrm{~h}$ of observing time. Unfortunately again, the particles that scatter the zodiacal light produce a strong infrared background and the proposed detector would register 150 photon $\mathrm{sec}^{-1}$ from this source. To measure the planetary signal against this large background to an accuracy of a few per cent would take a month.

The device proposed by Bracewell and MacPhie has two off-axis paraboloids producing the interference null. Tracking control uses the interferometer optics but at visual wavelengths, the infrared and visual beams being separated by 45 degree plates. The critical components of the detector will be kept at liquid or superfluid helium temperatures, less critical parts having to make do with the $30 \mathrm{~K}$ temperature of the triple point of neon.

Obviously this instrument is utilising all the hoped-for technical improvements. It is also apparent that all our attempts to detect non-solar planetary systems rely so far on the observation of minor fluctuations in large quantitites. We will have to wait a long time before we get a high definition view of another solar system, an agoinising wait which does little to stifle our feeling of loneliness in the universe.

\section{Reconstructing the past}

\section{from Peter D. Moore}

IT is the task of the palaeoecologist to make mental reconstructions of past environments on the basis of such evidence as assemblages of fossils. First the assemblage must be interpreted in terms of contemporaneous communities of organisms; second, the environment is reconstructed from what is known of the ecology of the organisms involved. The Quaternary palaeoecologist would seem to have certain advantages at both these interpretive stages as most of the species with which he deals are still extant and can therefore be subjected to various types of community analysis and other types of ecological enquiry. Just how real these advantages are may be questioned, for 